\title{
Amorphous silica at surfaces and interfaces: simulation studies
}

\author{
J. Horbach ${ }^{1)}$, T. Stühn ${ }^{1)}$, C. Mischler ${ }^{1)}$, W. Kob ${ }^{2)}$, and K. Binder ${ }^{1)}$ \\ 1) Institut für Physik, Johannes Gutenberg-Universität, \\ D-55099 Mainz, Staudinger Weg 7, Germany \\ 2) Laboratoire des Verres, Université Montpellier II, \\ F-34095 Montpellier, France
}

\begin{abstract}
The structure of surfaces and interfaces of silica $\left(\mathrm{SiO}_{2}\right)$ is investigated by large scale molecular dynamics computer simulations. In the case of a free silica surface, the results of a classical molecular dynamics simulation are compared to those of an ab initio method, the Car-Parrinello molecular dynamics. This comparative study allows to check the accuracy of the model potential that underlies the classical simulation. By means of a pure classical MD, the interface between amorphous and crystalline $\mathrm{SiO}_{2}$ is investigated, and as a third example the structure of a silica melt between walls is studied in equilibrium and under shear. We show that in the latter three examples important structural information such as ring size distributions can be gained from the computer simulation that is not accessible in experiments.
\end{abstract}

\section{Introduction}

The understanding of the structural and dynamic properties of silica at surfaces and interfaces is of special importance for technologically interesting systems such as semiconductor devices and nanoporous materials [1,2,3]. Furthermore, since amorphous silica surfaces are very reactive, they are used in catalysis and chromatography. Although there are different experimental methods such as infrared and Raman spectroscopy, atomic force microscopy and NMR to study surface properties, at least in the case of silica it is still difficult to make conclusive statements about the microscopic surface structure of $\mathrm{SiO}_{2}$ from experiments that use the latter methods [4, 5 , 6] 7 .

In order to shed more light on these issues, a molecular dynamics (MD) computer simulation is a very useful tool. In this method Newton's equations of motion are solved and thus one obtains the trajectories of the particles in the system from which one can calculate all the relevant quantities to characterize 
the microscopic structure (and dynamics). However, the predictive power of a MD simulation depends strongly on the quality of the potential with which one models the interactions between the particles. One possibility to check the accuracy of the model potential is a comparison to experiment. But, especially in the case of the surface structure of amorphous silica, there is a lack of experimental data. An alternative method to check the potential is presented in the next section: We use a combination of classical MD with a model potential and an $a b$ initio simulation technique, the so-called Car-Parrinello MD. The use of classical MD for the investigation of the non-bulk behavior of amorphous $\mathrm{SiO}_{2}$ is then presented for two other subjects, namely the structure of an interface between amorphous and crystalline $\mathrm{SiO}_{2}$ (Sec. 3) and the behavior of amorphous silica between two walls in equilibrium and under shear (Sec. 4). Finally we summarize the results in Sec. 5 .

\section{Free silica surfaces: $A b$ initio and classical molec- ular dynamics}

As a model to describe the interactions between the atoms in silica we use a pair potential which has been proposed by van Beest et al. 8]. It contains a Coulomb part and a short-ranged part,

$$
\phi(r)=\frac{q_{\alpha} q_{\beta} e^{2}}{r}+A_{\alpha \beta} \exp \left(-B_{\alpha \beta} r\right)-\frac{C_{\alpha \beta}}{r^{6}} \quad \alpha, \beta \in[\mathrm{Si}, \mathrm{O}],
$$

where $r$ is the distance between an atom of type $\alpha$ and an atom of type $\beta$. The effective charges are $q_{\mathrm{O}}=-1.2$ and $q_{\mathrm{Si}}=2.4$, and the parameters $A_{\alpha \beta}, B_{\alpha \beta}$, and $C_{\alpha \beta}$ can be found in the original publication. They were fixed by using a mixture of $a b$ initio calculations and classical lattice dynamics simulations. The long-ranged Coulomb forces (and the potential) were evaluated by means of the Ewald summation technique. As an integrator for the simulation we used a velocity Verlet algorithm [9] with a time step of 1.6 fs. More details on the potential and the calculation of the forces can be found in Ref. [10].

Although the BKS potential has turned out to be very good in the description of bulk properties of amorphous silica (see Ref. [1] and references therein), it is much less obvious that this potential is also reliable to model silica surfaces. The reason is that it was optimized to reproduce bulk properties such as the experimental elastic constants of $\alpha$ quartz. A similar fitting of the potential parameters to surface properties of real silica is difficult because of the lack of experimental data in this case. Although it is straightforward to investigate the properties of free surfaces of $\mathrm{SiO}_{2}$ by means of a MD using the BKS potential, it is not clear how one could test whether the results have anything in common with real silica.

One possibility to circumvent this problem is to use Car-Parrinello molecular dynamics (CPMD) 12 in which, different from a classical MD, the electronic degrees of freedom are taken into account via a density functional theory. Therefore, in contrast to a classical MD an effective potential between the ions 
is calculated self-consistently on the fly, i.e. the instantaneous geometry of the ions is always taken into account and one does not have to model the potential energy between the ions by a given function such as Eq. (11). However, it is not yet possible to replace the classical MD method by the CPMD, since due to the huge computational burden only relatively short time scales, a few ps, as well as small systems, a few hundred particles, can be simulated. In contrast to that classical MD allows one to simulate thousands of particles over several ns 9 .

Since the time scale which is accessible in a CPMD is very restricted the idea of our approach is to combine a classical MD and a CPMD (see also Ref. [13]). To this end, we first equilibrate the system with a classical MD in which we again model the interactions between the atoms by the BKS potential, Eq. (10). Then, we use these configurations as the starting point of a CPMD. The goal of this investigation is twofold: Firstly, we want to see whether the classical configurations are stable in CPMD. If this is the case we can subsequently compare the structural differences as obtained by the two methods. So we get an idea of how accurate the BKS potential is able to describe silica surfaces, and we have hints of how this potential energy model could be improved.

In order to investigate a free silica surface one could consider a film geometry, i.e. periodic boundary conditions (PBC) in two directions and an infinite free space above and below the remaining third direction. Unfortunately, this is not a very good solution since the Ewald summation technique for the long ranged Coulomb interactions becomes inefficient in this case 14. Therefore, we have adopted the following strategy which is illustrated in Fig. 1 i) We start with a system at $T=3400 \mathrm{~K}$ with $\mathrm{PBC}$ in three dimensions (box dimensions: $L_{x}=L_{y}=11.51 \AA$ and $L_{z}^{\prime}=23 \AA$ ). ii) We cut the system perpendicular to the $z$-direction into two pieces. This cut is done in such a way that we get only free oxygen atoms at this interface. iii) These free oxygen atoms are now saturated by hydrogen atoms. The place of these hydrogen atoms is chosen such that each of the new oxygen-hydrogen bonds is in the same direction as the oxygen-silicon bonds which were cut and have a length of approximately $1 \AA$. The interaction between the hydrogen and the oxygen atoms as well as the silicon atoms are described only by a Coulombic term. The value of the effective charge of the hydrogen atoms is set to 0.6 which ensures that the system is still (charge) neutral. iv) Atoms for which the distance from the interface that is less than $4.5 \AA$ are made immobile whereas atoms that have a larger distance can propagate subject to the force field. v) We add in $z$-direction an empty space $\Delta z=6.0 \AA$ and thus generate a free surface at around $14.5 \AA$. With this sandwich geometry we now can use periodic boundary conditions in all three directions. We have made sure that the value of $\Delta z$ is sufficiently large that the results do not depend on it anymore [10. Eventually, we have a system of 91 oxygen, 43 silicon, and 10 hydrogen atoms in a simulation box with $L_{x}=L_{y}=11.51 \AA$ and $L_{z} \approx 25 \AA$.

We have fully equilibrated 100 independent configurations for about $1 \mathrm{~ns}$. Using a subset of these configurations as starting points we subsequently started CPMD simulations [15]. We used conventional pseudopotentials for silicon and oxygen and the BLYP exchange functions [16 17]. The electronic wave-functions 
were expanded in a plane wave basis set with an energy cutoff of $60 \mathrm{Ry}$ and the equations of motion were integrated with a time step of 0.085 fs for 0.2 ps. More details on the CPMD simulations can be found in Ref. 10.

In the analysis of the CPMD run only those configurations were taken into account that were produced later than 5 fs after the start of the CPMD run in order to allow the system to equilibrate at least locally 13. From the 100 BKS configurations we have picked up those for which one of the three following cases holds: i) The surface exhibits no defects, i.e. all Si and $\mathrm{O}$ atoms are four and two-fold coordinated, respectively. ii) The surface has an undercoordinated oxygen atom and an undercoordinated silicon atom. iii) The surface has an overcoordinated oxygen atom and an undercoordinated silicon atom. We used two BKS configurations for each of the cases i)-iii) and started the CPMD runs. The computational effort for the latter was very large: To simulate $1 \mathrm{ps}$ one needs $11000 \mathrm{CPU}$ hours of single processor time (or about one month on 16 processors of a Cray T3E).

A quantity which is appropriate to characterize the network structure is the distribution of the ring size. A ring is defined as a closed loop of $n$ consecutive $\mathrm{Si}-\mathrm{O}$ segments. The largest differences between the results of the classical and that of the CPMD simulation is found for the short rings, i.e. $n<5$. Fig. 2 shows the probability to find a ring of size $n$ for the case of the BKS simulation and the CPMD. We see that with the BKS model the frequency with which a ring of size two occurs is overestimated by about a factor of two as compared to the CPMD result. In agreement with this observation we find that the overshoot in the $z$-dependence of the mass density profile near the surface is less pronounced for the CPMD than for the classical MD with the BKS potential (see inset of Fig. 2), since two-membered rings are relatively dense.

Another interesting result is the dependence of the distribution of angles $\mathrm{O}-\mathrm{Si}-\mathrm{O}$ on the ring size $n$ (Fig. 3). For large $n, n>4$, i.e. for rings which are normally found in the bulk, the results of the two different methods are in good agreement [13]. For smaller $n$, however, the mean $\mathrm{O}-\mathrm{Si}-\mathrm{O}$ angle from CPMD is shifted to larger values in comparison to that of the classical MD. This shift becomes more pronounced with decreasing $n$. Furthermore also the shape of the distributions starts to become different if $n$ is small.

This effect can be understood better if one analyzes the partial radial distribution functions $g(r)$ which are shown in Fig. 4 We see that for the $\mathrm{Si}-\mathrm{O}$ correlation the curves from the CPMD are shifted to larger distances by about $0.04 \AA$ and that this shift is independent of $n$. Also the $g(r)$ for the $\mathrm{O}-\mathrm{O}$ correlation are shifted to larger $r$, but now we observe different shifts for different $n$. In particular we note that the $\mathrm{O}-\mathrm{O}$ distance is nearly independent of $n$ for the CPMD whereas it increases with $n$ in the case of the BKS result. These effects result in the difference of the distribution of the $\mathrm{O}-\mathrm{Si}-\mathrm{O}$ angles if $n$ is small.

In conclusion we find that for the structure on larger length scales the BKS simulation and the CPMD yield similar results whereas the details of the structural elements on short scales (for instance, the short rings) are different in both methods. This shows that it is probably necessary to use ab initio methods like CPMD if one wants to reproduce quantitatively the properties of silica surfaces 
on short length scales.

\section{Interfaces between liquid and crystalline silica}

This section is devoted to a classical MD of an interface between liquid and crystalline silica where we have also used the BKS potential to model the interactions between the $\mathrm{Si}$ and $\mathrm{O}$ atoms. A straightforward method to produce the latter interface would be to wait for the formation of a crystalline nucleus in a supercooled melt. But presently this is an impossible task since the viscosity of a silica melt at the melting temperature, $T_{\mathrm{m}} \approx 2000 \mathrm{~K}$, is around $10^{7}$ Poise and thus the time scale that can be covered by a MD simulation is by far too short to observe the growth of a nucleus in a supercooled silica melt.

Our strategy to prepare a liquid-crystalline interface is as follows: In a first step a pure melt and a pure crystal ( $\beta$ cristobalite), both containing 1944 particles, are relaxed at $T=3100 \mathrm{~K}$ for $1.6 \mathrm{~ns}$ and $160 \mathrm{ps}$, respectively. Thereby, the box lengths are $L_{x}=L_{y}=21.375 \AA$ in $x$ and $y$ direction and $L_{z}=$ $61.465 \AA$ for the melt and $L_{z}=64.125 \AA$ for the crystal in $z$ direction. The corresponding densities are $2.32 \mathrm{~g} / \mathrm{cm}^{3}$ in the case of the melt and $2.22 \mathrm{~g} / \mathrm{cm}^{3}$ in the case of the crystal. In a second step 648 particles are removed from the equilibrated liquid configuration such that a free space is created in the middle of the simulation box. Then one cuts out a part of the crystal consisting of 648 particles that fits exactly in the latter free space (see Ref. 18] for the details) and one combines the liquid and crystalline pieces. This configuration is relaxed for about $30 \mathrm{ps}$ and as a result one obtains a system with liquid and crystalline $\mathrm{SiO}_{2}$ phases that are joined to each other via two interfaces. A snapshot of such

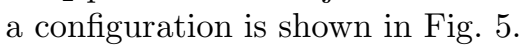

After the preparation procedure a microcanonical run is started where one expects that the crystal in the middle of the system melts because the temperature $T=3100 \mathrm{~K}$ is significantly above the melting temperature of our system [19]. A good order parameter to quantify the melting of the crystal is the intensity of the first Bragg peak in the static structure factor [18. The latter is shown in Fig. 6 for two different samples. Whereas in the first sample (upper plot of Fig. 6) the crystal does not melt at all even after about $2.6 \mathrm{~ns}$, in the same time span the second sample the crystal is completely melted. More details on that can be found in Ref. [18.

\section{A silica melt between walls}

We turn now our attention to a silica melt between walls to study the wall-fluid interface structure and to see how the melt behaves under shear. As a model to describe the interactions between the atoms in silica we use again the BKS potential. The walls were not constructed as to model a particular material but rather a generic surface that can be simulated conveniently. Each wall consisted of 563 point particles forming a rigid face-centered cubic lattice with a nearest-neighbor distance of $2.33 \AA$. These point particles interact with the 
atoms in the fluid according to a $12-10$ potential, $v(r)=4 \epsilon\left[(\sigma / r)^{12}-(\sigma / r)^{10}\right]$ with $\sigma=2.1 \AA, \epsilon=1.25 \mathrm{eV}, r$ being the distance between a wall particle and a $\mathrm{Si}$ or $\mathrm{O}$ atom. The main details of the MD simulations are as follows: The simulation box had linear dimensions $L_{x}=L_{y}=23.066 \AA$ in the directions parallel to the walls (in which also periodic boundary conditions were applied), and $L_{z}=31.5 \AA$ in the direction perpendicular to the walls. Thus, $N=1152$ atoms (384 $\mathrm{Si}$ atoms and $768 \mathrm{O}$ atoms) were contained in the system to maintain a density around $2.3 \mathrm{~g} / \mathrm{cm}^{3}$ which is close to the experimental one at ambient pressure. All runs were done in the NVT ensemble whereby the temperature was kept constant by coupling the fluid to a Nosé-Hoover thermostat 9]. We investigated the temperatures $T=5200 \mathrm{~K}, T=4300 \mathrm{~K}$, and $T=3760 \mathrm{~K}$ at which we first fully equilibrated the system for 29,65 , and $122 \mathrm{ps}$, respectively. At $T=5200 \mathrm{~K}$ and $T=3760 \mathrm{~K}$ we continued with additional runs over 164 and $490 \mathrm{ps}$, respectively, from which we analyzed the equilibrium structure. Then we switched on a "gravitational" field of strength $a_{e}=9.6 \AA / \mathrm{ps}^{2}$ that was coupled to the mass of the particles. With the acceleration field, runs were made over $736 \mathrm{ps}, 1.23 \mathrm{~ns}$, and $3.27 \mathrm{~ns}$ at $T=5200 \mathrm{~K}, 4300 \mathrm{~K}$ and $3760 \mathrm{~K}$, respectively. In addition at $T=5200 \mathrm{~K}$ we did a run over $1.72 \mathrm{~ns}$ with field strength $a_{e}=3.8 \AA / \mathrm{ps}^{2}$. The total amount of computer time spent for these simulations was 16 years of single processor time on a Cray T3E. More details on the simulation can be found in Ref. [20].

Fig. [7 shows the density profiles across half of the film for all atoms and for the oxygen and silicon atoms only. In contrast to typical density profiles in simple monoatomic liquids, the oscillations of the total profile, which indicate a layering near the walls, do not have a regular character. This behavior can be explained by the partial density profiles for oxygen and silicon. Obviously, the tetrahedra adjacent to the walls prefer to be aligned such that a two-dimensional plane forms which contains three out of the four oxygen atoms of a tetrahedron, as well as the silicon atom at their center (slightly further away from the wall), while the fourth oxygen atom of the tetrahedron has to be further away from the wall for geometrical reasons: this causes the second peak of the oxygen distribution. Thus, the walls have a tendency to "orient" the network of coupled $\mathrm{SiO}_{4}$ tetrahedra in the fluid locally. It is evident that the oscillations in the local density of both silicon and oxygen are rather regular, like a damped cosine function, but the wavelength and phase of both cosine functions are different: their superposition causes then the rather irregular layering structure of the $\mathrm{SiO}_{2}$ total density. We expect that similar effects also occur in many other associating molecular fluids confined between walls if the wall-fluid interaction is weak enough that it does not affect the chemical ordering in the fluid as it is the case in our system.

For $z>8 \AA$ the total density profile shows only small oscillations around a constant value of $2.3 \mathrm{~g} / \mathrm{cm}^{3}$ which is an indication for bulk behavior. Thus, keeping in mind that the density profile is symmetric, the bulk in our system seems to extend in $z$ direction from about $8 \AA$ to $23.5 \AA$, i.e. it has a width of about $15.5 \AA$. 
The behavior of the total density profiles at the temperatures $T=5200 \mathrm{~K}$ and $T=3760 \mathrm{~K}$ in equilibrium and with an external force with an acceleration of $9.6 \AA / \mathrm{ps}^{2}$ can be seen in Fig. $7 \mathrm{~b}$. In equilibrium the effect of decreasing temperature on the oscillations near the wall is an increase of the peak heights that is accompanied with a smaller value of $\rho(z)$ at the minima between the peaks. Thus, the layering becomes more pronounced if one decreases the temperature. If one switches on the gravitational-like field the effect is similar to an increase of the temperature. In the bulk region the density profiles are not very sensitive to a variation of temperature and/or the presence of the external force. Within the accuracy of our data the same value of about $2.3 \mathrm{~g} / \mathrm{cm}^{3}$ is reached for all four cases under consideration.

Fig. 8 8 a shows the ring distribution function $P(n)$ for $T=3760 \mathrm{~K}$ in the bulk and in two different wall layers denoted by WL1 and WL2. WL1 and WL2 are defined as the regions which are respectively within a distance of $6.25 \AA$ and $3.0 \AA$ away from the wall corresponding to the second minimum of the density profile for silicon and the first minimum of the total density profile, respectively (see Fig. 7 ). In each region, i.e. bulk, WL1, and WL2, we took only those rings into account that fit completely into it. Thus, in WL2 those rings are counted that are formed at each case by the first and the second $\mathrm{O}$ and Si layers (with respect to the distance from the wall), whereas with WL2 only those rings are taken into account that are formed by the first $\mathrm{O}$ and the first $\mathrm{Si}$ layer. This is justified because the first two oxygen and the first two silicon layers are welldefined in that the minima in the corresponding density profiles are close to zero density in the case of WL2 and around the small value $\rho=0.5 \mathrm{~g} / \mathrm{cm}^{3}$ in the case of WL1. Furthermore, we can infer from Fig. (7) that in contrast to the second layers the first oxygen layer overlaps strongly with the first silicon layer and the overall thickness of both layers is only about $2 \AA$. Thus, the first oxygen and the first silicon layer form a quasi-two-dimensional plane and $P(n)$ for WL2 gives a distribution of rings that have an orientation parallel to the walls whereas in $P(n)$ for WL1 also the rings perpendicular to the walls are included.

In bulk simulations of $\mathrm{SiO}_{2}$ one finds a maximum around $n=6$ [21]. This is plausible since in silica the high-temperature crystalline phase at zero pressure, $\beta$-cristobalite, exhibits only six-membered rings. In WL1 the probability for $n \geq 6$ is smaller than in the bulk in favor of a relatively high probability of $n=3$ and $n=4$. In WL2 it is the other way round: $n=4$ and even more $n=5$ are less frequent than in the bulk in favor of $n=8,9,10$. The ring structure near the walls that corresponds to these findings is as follows: Perpendicular to the walls small rings with $n=3,4$ are seen such that, e.g., $n=3$ is formed by two silicon atoms from the first silicon layer with a third one from the second silicon layer. In contrast to that, parallel to the walls (considering the first oxygen and the first silicon layer) an open structure with relatively large rings is observed which compensates somewhat the dense packing of $\mathrm{SiO}_{4}$ tetrahedra perpendicular to the walls.

Fig. 8 $\mathrm{b}$ shows the behavior of $P(n)$ in the bulk at the two temperatures $T=$ $3760 \mathrm{~K}$ and $T=5200 \mathrm{~K}$ in equilibrium and under shear. We can immediately 
infer from the figure that the considered shear fields have only a small effect on the structure. At $T=5200 \mathrm{~K}$ one has a relatively large amount of two- and three-membered rings and their frequency is more than a factor of two smaller at $T=3760 \mathrm{~K}$. In Ref. 21] it was shown that their frequency of occurrence decreases further with decreasing temperature such that the amount of twomembered rings falls far below $1 \%$ for systems that have typical structural relaxation times of the order of $1 \mathrm{~ns}$.

In contrast to the bulk in WL1 significant changes in the ring distribution take place if the system is sheared (see Fig. 8 8 ), and the external force field affects the ring structure such that small and large rings are formed, while at the same time especially the amount of six-membered rings decreases. Only for the smaller field strength $a_{\mathrm{e}}=3.8 \AA / \mathrm{ps}^{2}$ there are no significant changes in the ring distribution. As we have shown elsewhere 20 the latter is accompanied with a very small slip motion at the walls whereas a large slip velocity is correlated with strong rearrangements in the ring structure. One can also infer the remarkable fact from Fig. 88 that the probability to find rings with $n=3,4$ does not change very much when an external acceleration field is switched on. This is reasonable because these small-membered rings, as we have seen before, are located perpendicular to the walls and thus they are very stable to shear forces that are imposed parallel to the walls.

The strongest rearrangements in the ring structure due to a shear field are found when we consider the region WL2. The corresponding curves are shown in Fig. 8 8 . Again, there are only minor changes in $P(n)$ at $T=5200 \mathrm{~K}$ and $a_{\mathrm{e}}=3.8 \AA / \mathrm{ps}^{2}$ as compared to the corresponding equilibrium case which is, as mentioned before, related to the presence of only a very small slip velocity. For the higher acceleration field, $a_{\mathrm{e}}=9.6 \AA / \mathrm{ps}^{2}$, the ring structure becomes more heterogeneous and the effect of the external field is if one would locally increase the temperature. The rearrangements in the ring structure can be summarized as follows: Rings mainly of size $n=6,7,8$ are broken under the influence of the shear force and instead small rings with $n<4$ and very large rings with $n \geq 9$ are formed.

\section{Summary}

We have shown that large scale MD simulations are able to give a lot of insight into the microscopic surface and interface structure of silica. In all three examples that we have presented in this report it is necessary to simulate relatively large systems on a relatively large time scale and thus the use of parallel supercomputers is indispensable. In our study of the free silica surface the CarParrinello MD was used for which length and time scales that can be covered are even very restricted on a parallel computer (CPMD simulations are typically on a ps time scale for systems of about 100 particles). Thus, the development and application of more powerful parallel computers is required to gain further insight from atomistic simulations.

Acknowledgments: We thank the HLRZ Stuttgart for a generous grant of 
computer time on the CRAY T3E. C. M. and T. S. are grateful to SCHOTT Glas for partial financial support.

\section{References}

[1] A. P. Legrand, The Surface Properties of Silica (Wiley, New York, 1998).

[2] R. K. Iler, The Chemistry of Silica (Wiley, New York, 1979).

[3] C. R. Helms and B. D. Deal, The Physics and Chemistry of $\mathrm{SiO}_{2}$ and the $\mathrm{Si}-\mathrm{SiO}_{2}$ Interface (Plenum, New York, 1993).

[4] A. Grabbe, T. A. Michalske, and W. L. Smith, J. Phys. Chem. 99, 4648 (1995).

[5] P. K. Gupta, D. Inniss, C. R. Kurkjian, and Q. Zhong, J. Non-Cryst. Sol. 262, 200 (2000).

[6] J.-F. Poggemann, A. Go, G. Heide, E. Rädlein, and G. H. Frischat, J. Non-Cryst. Sol. 281, 221 (2001).

[7] I.-S. Chuang and G. F. Maciel, J. Am. Chem. Soc. 118, 401 (1996).

[8] B. W. van Beest, G. J. Kramer, and R. A. van Santen, Phys. Rev. Lett. 64 1955 (1990).

[9] K. Binder, J. Horbach, W. Kob, W. Paul, and F. Varnik, J. Phys.: Condens. Matter 15 (2003).

[10] C. Mischler, Molekulardynamik-Simulation zur Struktur von $\mathrm{SiO}_{2}-$ Oberflächen mit adsorbiertem Wasser, Ph.D. thesis, Mainz 2002; C. Mischler, W. Kob, and K. Binder, Comp. Phys. Comm. 147, 222 (2002).

[11] J. Horbach and W. Kob, Phys. Rev. B 60, 3169 (1999).

[12] R. Car and M. Parrinello, Phys. Rev. Lett. 55, 2471 (1985).

[13] M. Benoit, S. Ispas, P. Jund, and R. Jullien, Eur. Phys. J. B 13, 631 (2000).

[14] A. Arnold, J. de Joannis, and C. Holm, J. Chem. Phys. 117, 2496 (2002); J. de Joannis, A. Arnold, and C. Holm, J. Chem. Phys. 117, 2503 (2002).

[15] CPMD Version 3.3a, J. Hutter, P. Ballone, M. Bernasconi, P. Focher, E. Fois, S. Goedecker, M. Parrinello, and M. Tuckermann, MPI für Festkörperforschung and IBM Research 1990-2000.

[16] N. Trouiller and J. L. Martins, Phys. Rev. B 43, 1993 (1991).

[17] C. Lee, W. Yang, and R. G. Parr, Phys. Rev. B 37, 785 (1988).

[18] T. Stühn, Ph.D. thesis, Mainz, 2003. 
[19] A. Roder, W. Kob, and K. Binder, J. Chem. Phys. 114, 7602 (2001).

[20] J. Horbach and K. Binder, J. Chem. Phys. 117, 10798 (2002).

[21] K. Vollmayr, W. Kob, and K. Binder, Phys. Rev. B 54, 15808 (1996). 

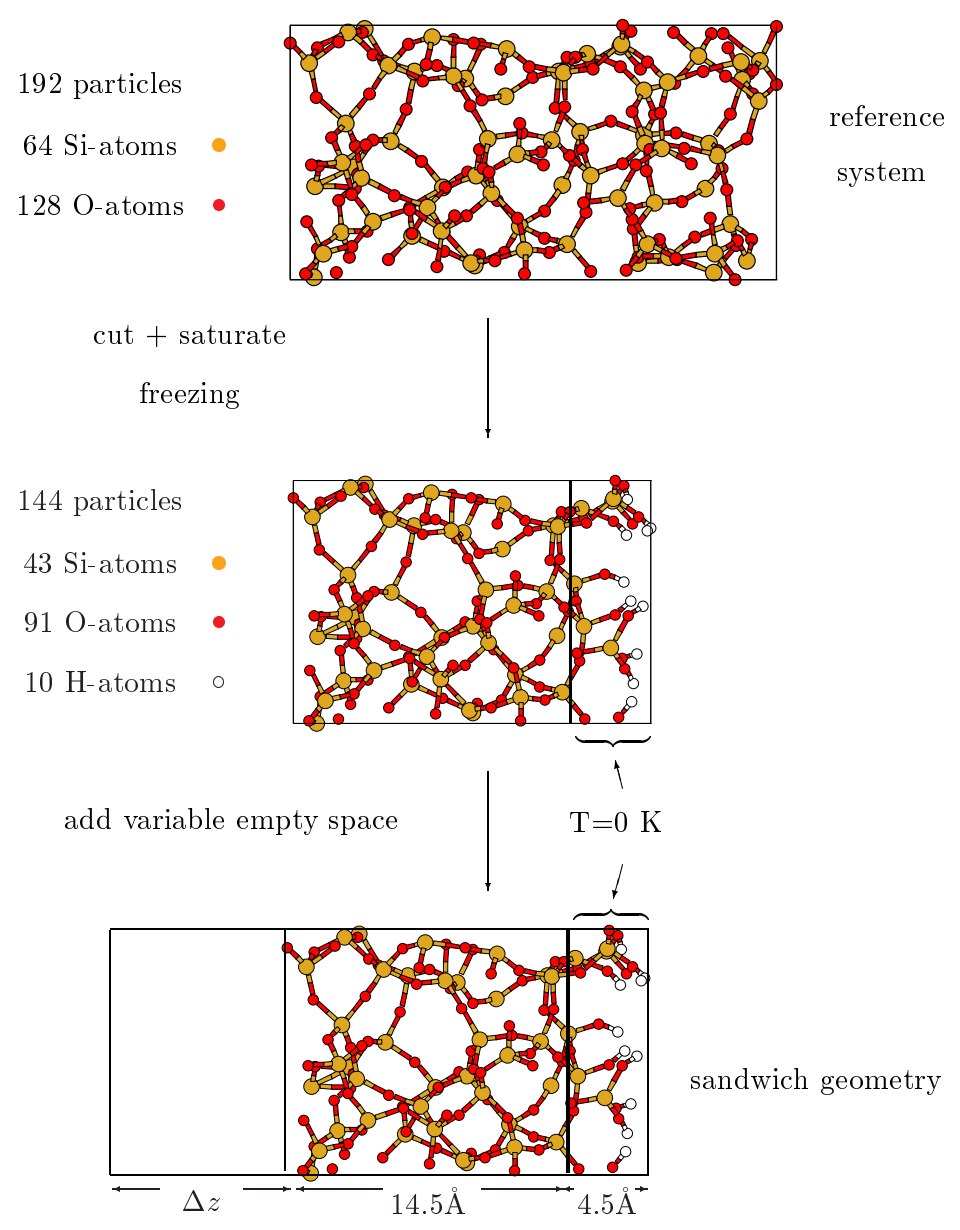

Figure 1: Procedure to create the used sandwich geometry. 


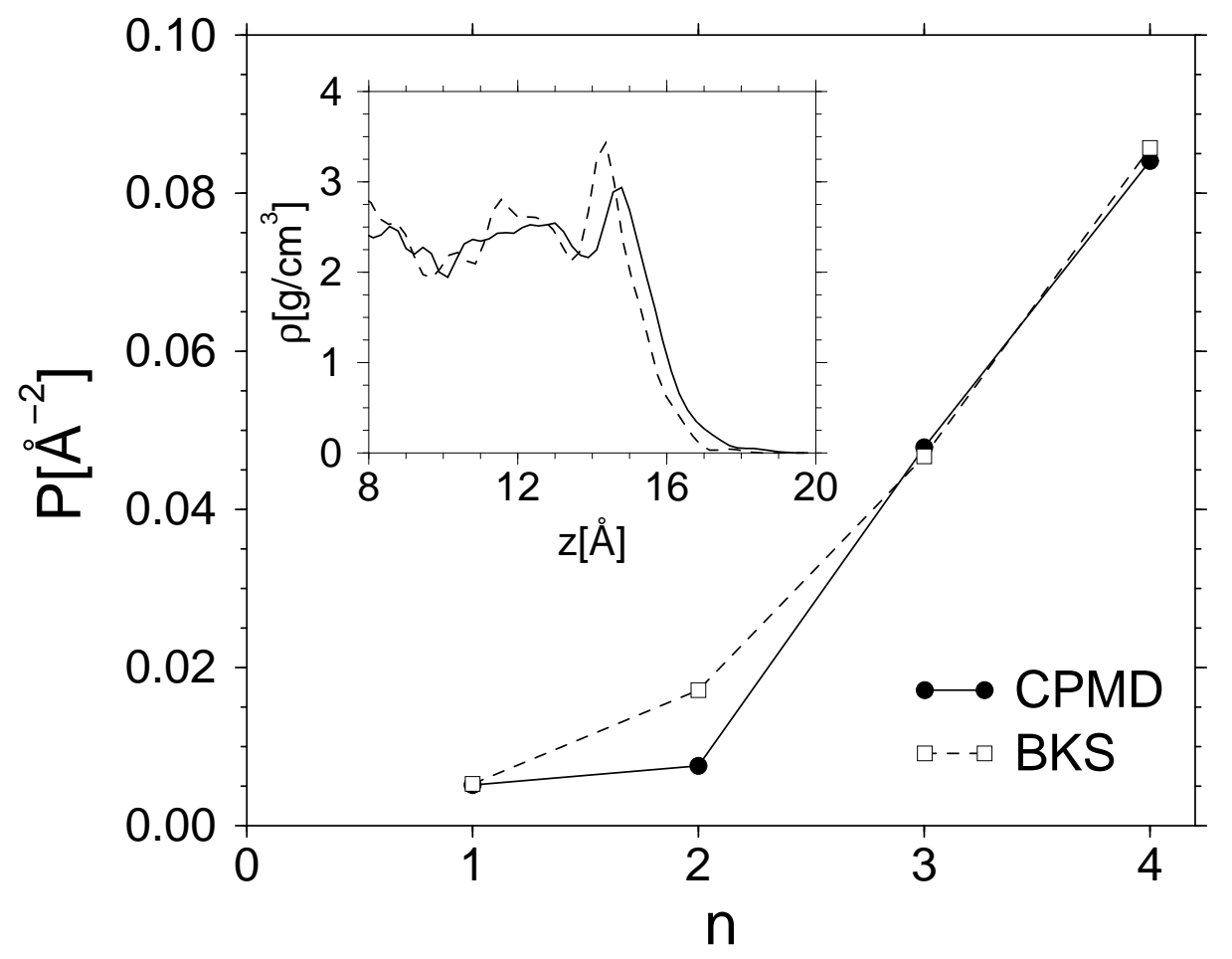

Figure 2: Probability to find a ring of size $n$. Inset: $z$-dependence of the mass density. 


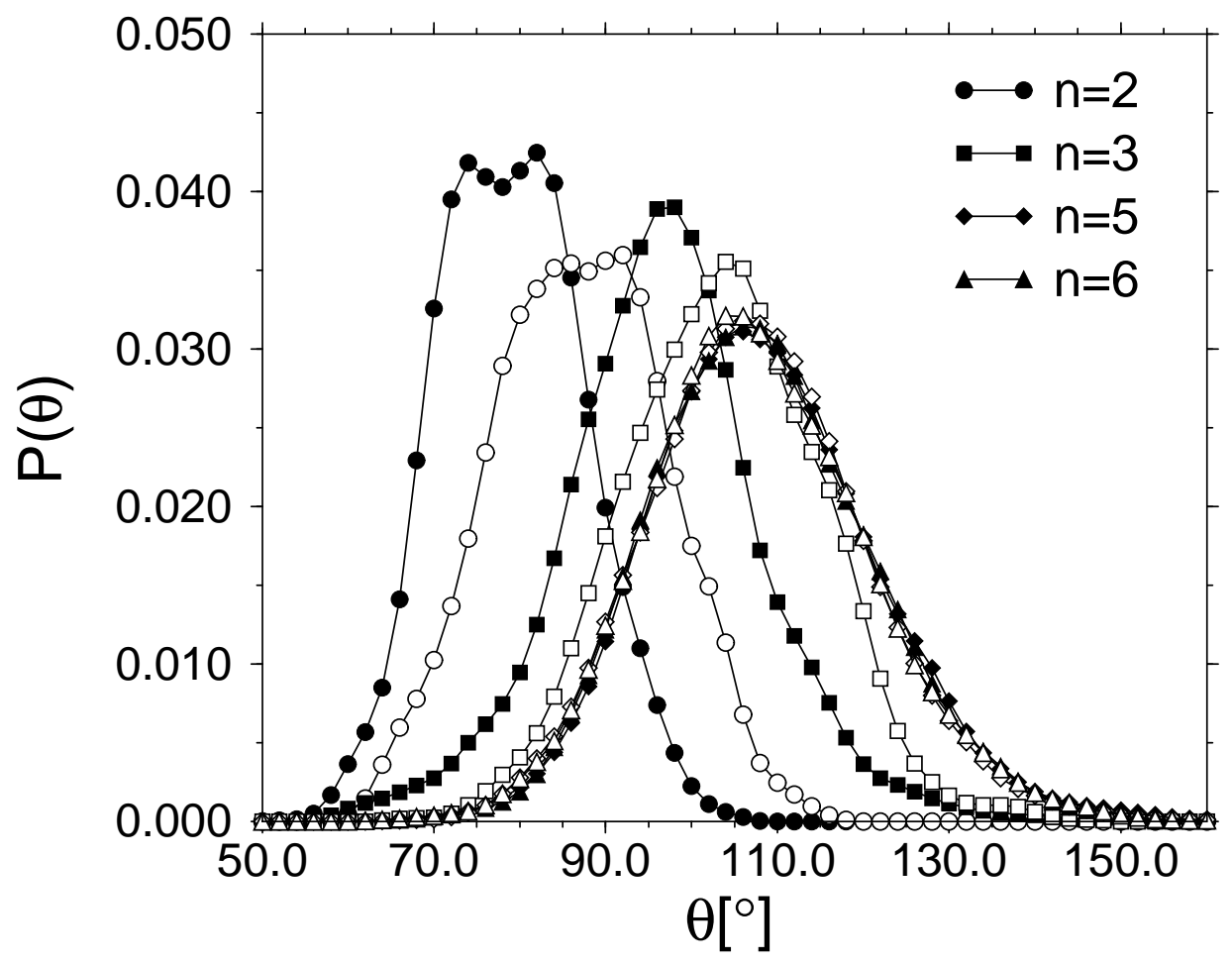

Figure 3: Distribution of $\mathrm{O}-\mathrm{Si}-\mathrm{O}$ angles for different ring sizes (BKS: filled symbols, CPMD: open symbols). 

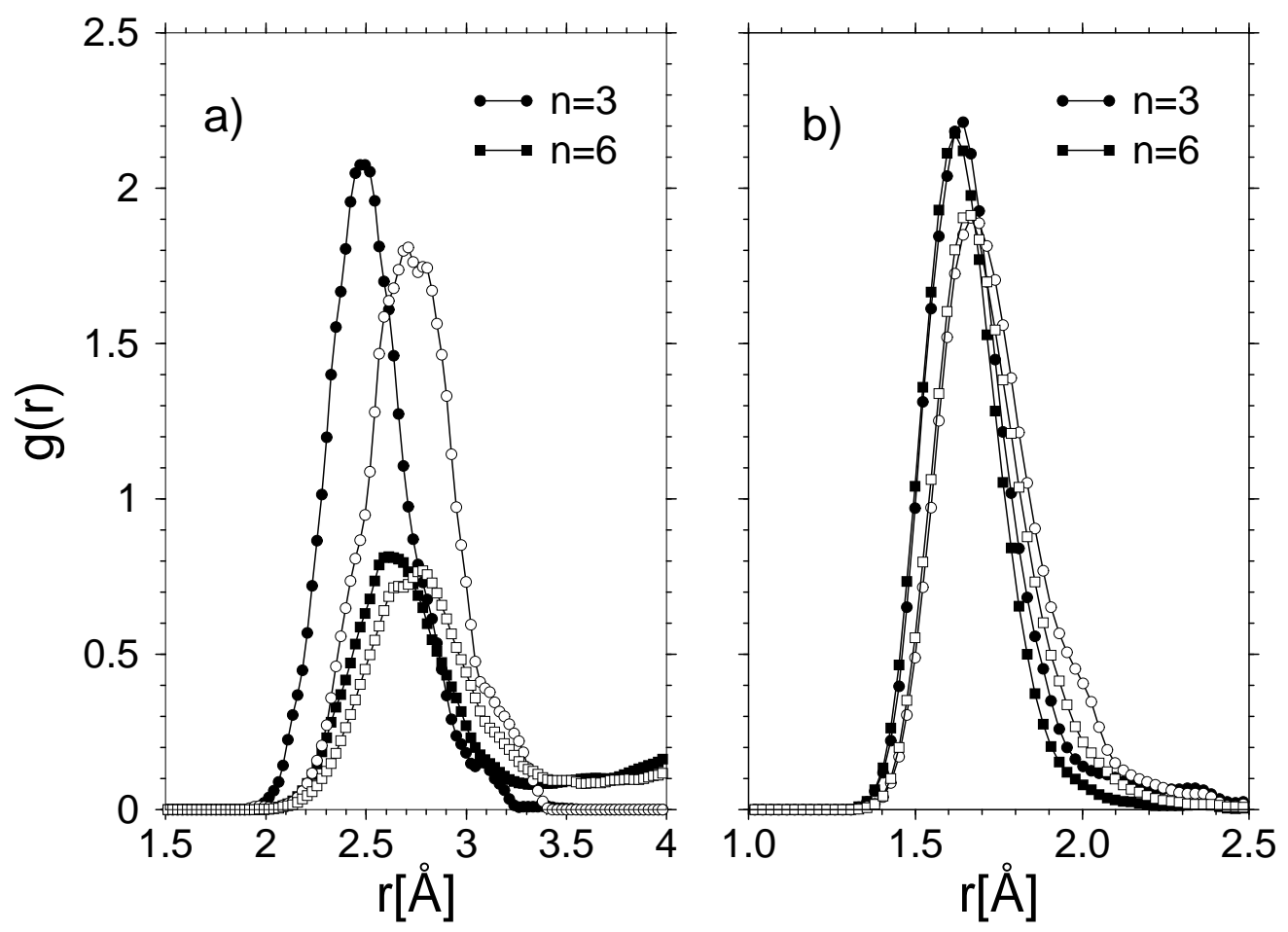

Figure 4: Radial distribution function for different ring sizes, (a) O-O and (b) $\mathrm{Si}-\mathrm{O}$ (BKS: filled symbols, CPMD: open symbols). 


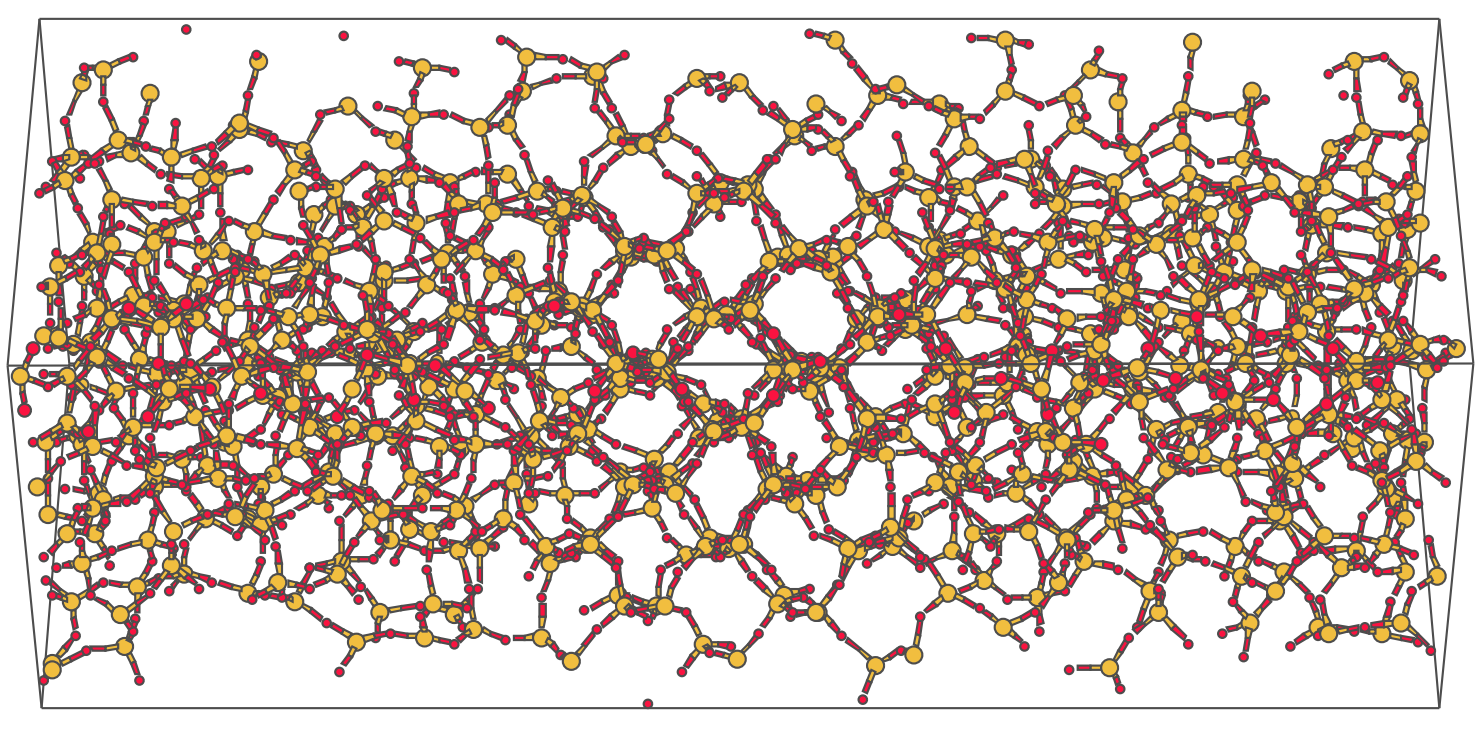

Figure 5: Snapshot of the system at $T=3100 \mathrm{~K}$. 

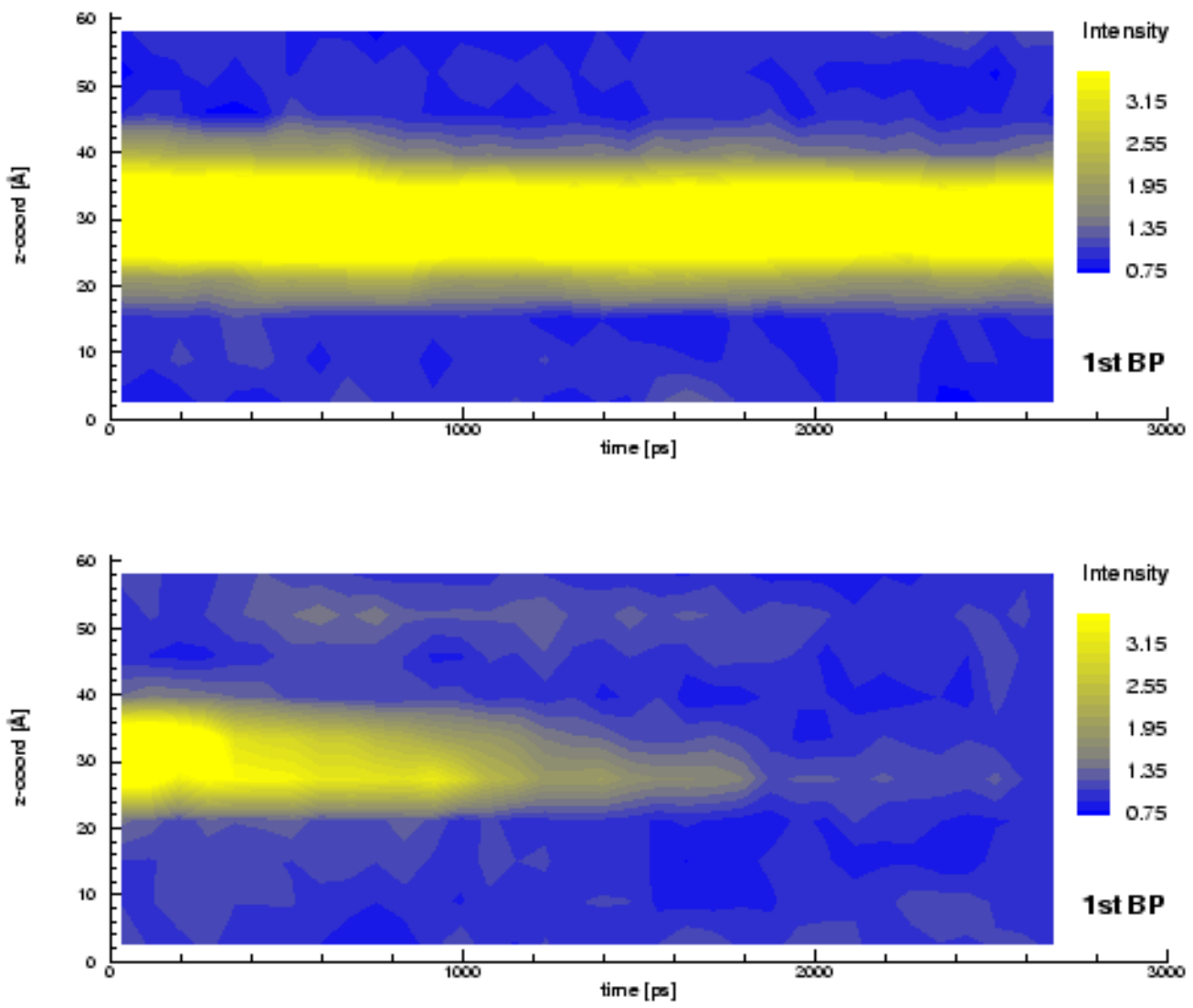

Figure 6: Time evolution of the intensity of the first Bragg peak in the static structure factor for two different samples at the temperature $T=3100 \mathrm{~K}$. 

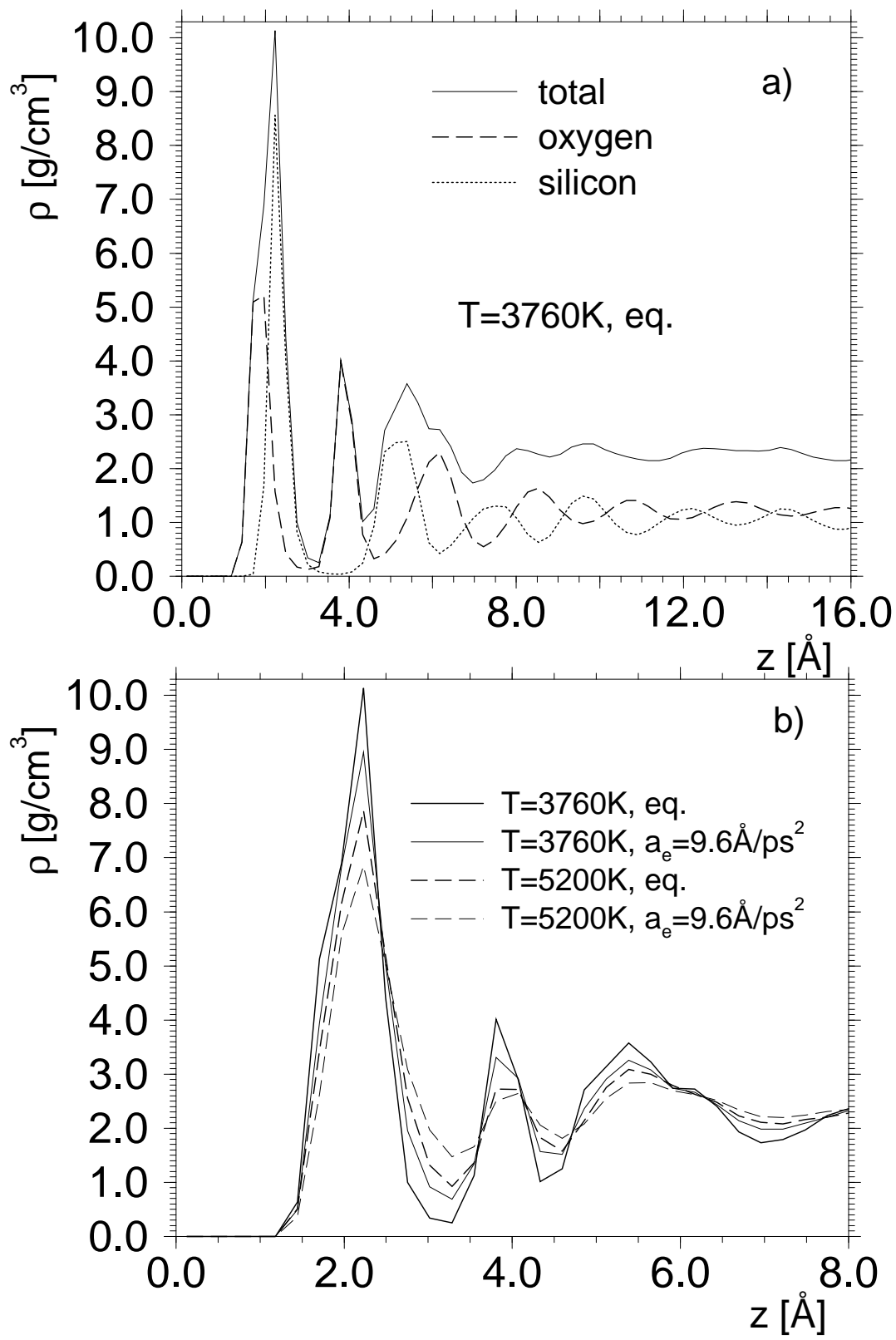

Figure 7: (a) Total density profile and partial density profiles for oxygen and silicon in a system at equilibrium at $T=3760 \mathrm{~K}$. (b) Total density profiles at equilibrium and with acceleration field $a_{e}=9.6 \AA / \mathrm{ps}^{2}$ for the indicated temperatures. 

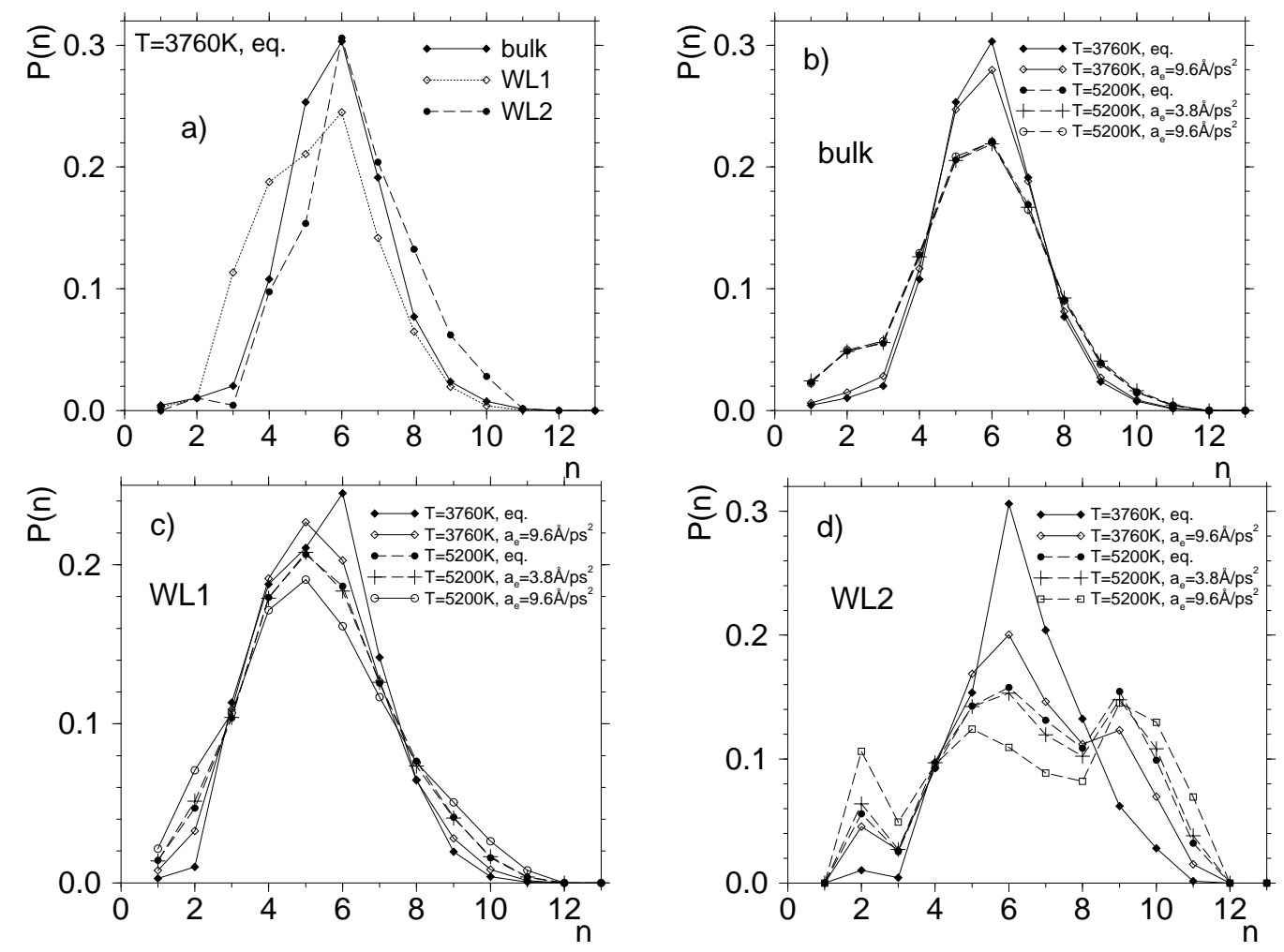

Figure 8: Probability $P(n)$ that a ring has a length $n$, a) at $T=3760 \mathrm{~K}$ in equilibrium for the bulk, WL1, and WL2, b) at $T=3760 \mathrm{~K}$ and $T=5200 \mathrm{~K}$ in equilibrium and under the indicated acceleration fields in the bulk, c) the same as in b) but for WL1, and d) also the same as in b) but for WL2. See text for the definition of WL1 and WL2. 